\title{
TENDENCIAS EN LOS ESTUDIOS SOBRE COMUNICACIÓN NO VERBAL: CONVERGENCIAS Y DIVERGENCIAS ${ }^{1}$
}

\section{TRENDS IN STUDIES ON NON-VERBAL COMMUNICATION: CONVERGENCES AND DIVERGENCES}

\author{
Pablo Sánchez Abascal \\ Universidad Autónoma de Madrid \\ pablo.sanchezabascal@estudiante.uam.es
}

\section{Resumen:}

Este estudio revisa diez trabajos desarrollados en el área de la comunicación no verbal llevados a cabo en los últimos cinco años desde el comienzo de nuestra investigación doctoral en el año 2017. Para tal fin, se delimitan las tendencias temáticas de los trabajos, los objetivos de estudio y las metodologías que se aplican. Las conclusiones de la revisión se recogen en el apartado de Convergencias y divergencias en el que, entre otros resultados, se evidencia la predominante presencia de los análisis quinésicos sobre el resto de los sistemas no verbales, el marcado carácter interdisciplinar de los estudios, o la interdependencia relacional entre el código verbal y el contexto de la interacción comunicativa.

Palabras clave: Comunicación no verbal, Sistema quinésico, Interacción comunicativa.

\begin{abstract}
:
This study reviews ten of the last investigations developed in the area of non-verbal communication in the last five years since the beginning of our doctoral research in 2017. In order to reach this target, this analyze delimits trends in studies on non-verbal communication, their studies objectives and their methodologies. The conclusions of this study are included in section Convergences and Divergences in which, among other results, the predominant role of the kinetic analysis over the rest of the nonverbal systems, the deeper interdisciplinary nature of the nonverbal studies or their relational interdependence are evidenced between the verbal code and the context of the communicative interaction.
\end{abstract}

Keywords: Non-verbal communication, Kinesics system, Communicative interaction.

Recibido: 21 de febrero de 2021

Aceptado: 04 de junio de 2021

\footnotetext{
${ }^{1}$ Artículo derivado del proyecto "El uso funcional de la mirada en las interacciones comunicativas L1 y L2 en hispanohablantes peninsulares y suecos" perteneciente al plan 646- del programa de Doctorado en Estudios Hispánicos, Lengua, Literatura, Historia y Pensamiento, Universidad Autónoma de Madrid (UAM), con identificación $\mathrm{N}^{\circ} 351808$, codirigida por el Departamento de Lenguas Clásicas y Romanas de la Universidad de Estocolmo.
} 
Artículo. Pablo Sánchez Abascal. "Tendencias en los estudios sobre comunicación no verbal: convergencias y divergencias."

\section{Antecedentes}

Los estudios desarrollados en el área de comunicación no verbal (en adelante, CNV) han atravesado diferentes fases desde que, en el año 1872, el investigador y antropólogo Charles Darwin escribiera The Expression of the Emotions in Man and Animals, obra considerada como el primer estudio sobre la expresión facial de las emociones en seres humanos y animales. Sin embargo, fue el propio Darwin quien indicó la existencia de estudios no verbales anteriores a su obra citando para ello a psicólogos, filósofos, anatomistas, dibujantes e investigadores como fuente de inspiración para la redacción de su trabajo en las expresiones de seres humanos, sobre todo de niños y animales.

El listado de autores a los que Darwin se refiere en su obra es tan amplio como diversos son los campos de estudio con los que estos se relacionan. Ejemplo de ello son las ilustraciones del pintor Louise Pierre Gratiolet (1865) o los ensayos y las obras sobre expresiones de los sentimientos en infantes y animales de Herbert Spencer (1855), lo que indica que el interés por el conocimiento y la profundización en el significado de los signos no verbales en los procesos de interacción comunicativa ha estado presente en los estudios de diferentes disciplinas desde hace mucho tiempo. Asimismo, y aunque desde la elaboración de los primeros trabajos sobre el estudio de la CNV hayan pasado ya varios siglos, todavía hoy se pueden encontrar investigaciones recientes que se enfocan en la aplicación práctica de los signos no verbales y culturales en la interacción lingüística y en la enseñanza de lenguas extranjeras. Entre ellos, se pueden destacar las investigaciones realizadas por investigadores como Birdwhistell (1970), Ekman y Friesen (1969, 1986), Davis (1973), Knapp (1980) o los múltiples estudios desarrollados por Cestero Mancera $(1998,1999,2004)$ y Poyatos (1994a, 1994b, 2017).

Más próximos al marco temporal de este artículo, y con el objetivo de delimitar la orientación práctica de los últimos estudios sobre CNV en el área de la investigación sociocultural, se han analizado diez trabajos desarrollados en los últimos cinco años desde el comienzo de nuestra investigación en el año 2017. Dichos trabajos -artículos de investigación que aparecen en revistas especializadas de Lingüística Aplicada, tesis doctorales y trabajos fin de máster-, afrontan el proceso de investigación de los signos no 
verbales desde perspectivas de estudio diferentes, aunque complementarias, que enriquecen de esta forma el valor práctico de este trabajo.

\section{Criterios de selección de los estudios}

Los criterios que rigen la selección de los estudios que aparecen en este trabajo se basan en las recomendaciones de autores especializados en la búsqueda de fuentes de información para trabajos científicos como Núñez (2002) o Espinoza (2003). De entre sus aplicaciones, y en base a las recomendaciones que también se recogen en el trabajo de Perdomo (2008), queremos destacar los siguientes principios de selección:

\begin{tabular}{|c|c|}
\hline & $\begin{array}{l}\text { Se seleccionan artículos de investigación dirigidos a una audiencia } \\
\text { académica que se pueden encontrar en revistas especializadas en } \\
\text { versión electrónica o páginas gubernamentales en las cuales se } \\
\text { publican las ponencias arbitradas en extenso (Perdomo, 2008). }\end{array}$ \\
\hline Sitio & $\begin{array}{l}\text { Se seleccionan artículos de investigación que provengan de } \\
\text { organismos gubernamentales, sitios académicos de universidades de } \\
\text { cualquier parte del mundo, bibliotecas virtuales, sociedades científicas } \\
\text { y revistas electrónicas arbitradas (Perdomo, 2008). }\end{array}$ \\
\hline Tex & $\begin{array}{l}\text { Se seleccionan artículos de investigación o estudios científicos que, o } \\
\text { bien forman parte de publicaciones científicas periódicas, o bien son } \\
\text { publicaciones en línea de tesis de postgrado que han sido previamente } \\
\text { revisadas por un jurado (Perdomo, 2008). }\end{array}$ \\
\hline Temporalidad & $\begin{array}{l}\text { Se seleccionan artículos de investigación o estudios científicos } \\
\text { realizados desde el año } 2012 \text { en adelante, es decir, en el lapso de los } \\
\text { últimos cinco años desde el comienzo de nuestra investigación. }\end{array}$ \\
\hline Otros factores & $\begin{array}{l}\text { La originalidad en la temática, la estructuración y la organización del } \\
\text { trabajo, la utilidad en relación con el estudio de los signos no verbales } \\
\text { y el alcance de la información. }\end{array}$ \\
\hline
\end{tabular}

Cuadro 1. Criterios de selección 
Artículo. Pablo Sánchez Abascal. "Tendencias en los estudios sobre comunicación no verbal: convergencias y divergencias.”

\section{Análisis de los estudios}

\subsection{El componente no verbal en el aula de ELE. Consideraciones para su aprendizaje significativo e intercultural a partir de WebQuest (2006)}

Artículo publicado en la revista Nebrija de Lingüística Aplicada a la Enseñanza de las Lenguas en el que Méndez Guerrero analiza el papel del componente no verbal en la enseñanza del español como lengua extranjera calificando el signo no verbal como un elemento cultural de la comunicación humana y encuadrando su estudio en el área de la competencia sociocultural y dentro de la competencia intercultural. Este artículo destaca la importancia de estudiar el componente no verbal de la lengua desde una posición intermedia entre la lengua meta y la lengua de estudio relacionando la forma, el uso o el funcionamiento de los signos no verbales de ambas lenguas. Tal y como se destaca en este artículo, Dago García (2008) avala el tratamiento implícito de la cultura en el aula de español como lengua extranjera (en adelante, ELE) recomendando la nivelación de los contenidos en base a su funcionalidad, frecuencia y dificultad.

Méndez Guerrero recoge un principio didáctico fundamental para la enseñanza de los signos no verbales en lenguas extranjeras: partir de los conocimientos previos de la cultura de los estudiantes para, paulatina y progresivamente, generar conocimiento sobre sus cimientos. Para ello, y refiriéndose a la estructura de trabajo piramidal de Moreno de los Ríos (1998), se presentan las que se consideran las tres fases de aprendizaje:

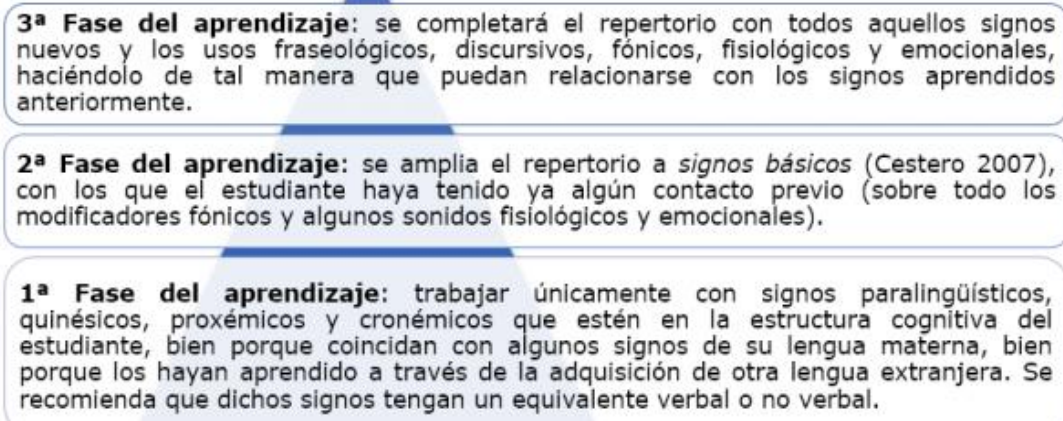

Figura 1. Esquema para el aprendizaje significativo de la CNV en el aula de ELE. 
Según Méndez Guerrero, los recursos que se pueden utilizar para la enseñanza de los signos no verbales son múltiples y variados, si bien, de entre todos ellos, se recomienda la elaboración de repertorios lingüísticos/culturales o de inventarios de signos no verbales. Méndez Guerrero sugiere también la aplicación didáctica de los contenidos no verbales mediante el uso de herramientas virtuales y asociadas a las nuevas tecnologías (TICs) destacando la funcionalidad práctica de las denominadas WebQuest. Para la secuencia de trabajo en dichas WebQuest se recomiendan las siguientes indicaciones:

a. Tomar conciencia de las conductas basadas en la identidad cultural entendiendo sus valores, ideas y actitudes que nos diferencian de otros grupos sociales.

b. Tomar conciencia de las conductas basadas en la cultura de los otros, asumiendo el importante valor del reconocimiento de la diferencia.

c. Ser capaz de explicar los propios posicionamientos culturales que, en ningún caso, se deben afirmar por la negación de los posicionamientos de los demás.

En su parte final, el trabajo de Méndez Guerrero hace referencia al proceso de adquisición de los signos no verbales en el estudio de ELE a través de un aprendizaje significativo mediante una reconciliación integradora entre los conocimientos previos de los estudiantes y los nuevos de la cultura en la que se sumergen. Para ello, se insta a los profesores tener en cuenta las consideraciones necesarias para la progresión de los contenidos que se presentan en el aula en base a las necesidades de los estudiantes.

\subsection{La interpretación del silencio en la interacción. Principios pragmáticos, cognitivos y dinámicos (2016)}

Artículo publicado en la revista Pragmalingüística de la Universidad de Cádiz en el que, de nuevo la investigadora Méndez Guerrero, analiza el funcionamiento de los signos no verbales en las interacciones comunicativas conversacionales. Para ello, analiza el condicionamiento que ejercen los factores extralingüísticos en dichas interacciones centrando su atención en el papel que juega el silencio en los procesos inferenciales de la 
Artículo. Pablo Sánchez Abascal. "Tendencias en los estudios sobre comunicación no verbal: convergencias y divergencias."

interpretación de un mensaje. De esta manera, Méndez Guerrero señala que el proceso inferencial que llevan a cabo los hablantes para interpretar el signo paralingüístico del silencio está regulado por una serie de principios básicos que son constantes. También señala que la comunicación es un proceso continuo que, aunque pueda producir momentos de no actividad en el canal verbal, tiene siempre lugar en el canal no verbal. De hecho, el silencio conversacional aporta un significado pragmático durante el proceso ostentivo-inferencial ${ }^{2}$ y puede utilizarse con otros elementos lingüísticos.

El trabajo de Méndez Guerrero pone de manifiesto que, en el caso del silencio, el papel del oyente es especialmente activo para la comprensión de la intención comunicativa del emisor. Y a pesar de que se ha dicho del silencio que es un signo vago e impreciso (Terrón Blanco, 1992), la ambigüedad intrínseca que se atribuye a los silencios queda minimizada una vez que este viene acompañado de una situación o un contexto que lo delimita. Desde un punto de vista pragmático, Méndez Guerrero señala la existencia de dos tipos fundamentales de silencio: los silencios que poseen un valor meramente de signos como marcadores discursivos, estructurales, psicológicos o normativos en la interacción, y los silencios que, además de su propia condición, son elegidos opción lingüística y tienen un valor de acción en la construcción activa del mensaje.

Méndez Guerrero presenta diferentes definiciones del concepto de silencio en el ámbito de la pragmática y considera que, si la interacción comunicativa tiene lugar mediante el acto silencioso, precisará del contexto en mayor medida que cuando se usa la palabra. Además, se resalta el concepto de que el empleo del silencio conlleva implicaturas con diferentes niveles de complejidad en su interpretación y destaca que en el proceso de interpretación pragmática del significado del silencio se debe atender al carácter dinámico del signo y a la relación que se establece con otros signos verbales y no verbales que lo preceden o que aparecen junto al silencio, con el contexto (situacional y sociocultural), con la relación social de los participantes o con los conocimientos previos y compartidos y los procesos cognitivos.

A modo de conclusión, el artículo de Méndez Guerrero resalta la necesidad una teoría que conciba y explique los actos silenciosos en un contexto más amplio de acción, es decir,

\footnotetext{
${ }^{2}$ Modelo interaccional en el que la comunicación se consigue mediante la producción y la interpretación de pruebas o evidencias. Como señalan Sperber y Wilson (195): «El emisor produce un estímulo que hace mutuamente manifiesto para sí mismo y para el oyente el cual, mediante dicho estímulo, hace más manifiesto un conjunto de supuestos».
} 
como parte de un discurso conectado y cambiante en el que se puedan interpretar todos los elementos que interactúan en los procesos de interacción comunicativa.

\subsection{The role of Non-verbal communication in English Teaching (2015)}

Artículo publicado en la revista digital Jurnal Transformasi en el que Sutiyatno analiza el papel que juegan los signos no verbales en el proceso de enseñanza del inglés como lengua extranjera (en adelante, LE). Asimismo, Sutiyatno estudia en este trabajo la relación que se establece en el aula entre estudiantes y profesores entendiendo a estos últimos como un puente de entrada y de intercambio cultural para el desarrollo del proceso -consciente o inconsciente- de la comunicación intercultural de los estudiantes. De esta manera, sostiene que el canal comunicativo no verbal carece de una estructura formal rígida y que los signos no verbales se reconocen, fundamentalmente, a través de las normas culturales, el bagaje cultural y socioeconómico, la educación, el género, la edad o la idiosincrasia personal. Sutiyatno añade, además, que los verdaderos desencuentros comunicativos tienen lugar no tanto cuando se producen malentendidos verbales sino cuando no se entiende el valor que toman los silencios en los procesos de interacción comunicativa. Se resalta también que la comunicación no verbal se caracteriza por operar en el momento presente, es decir, en la situación y el contexto en el que tiene lugar la interacción comunicativa, haciendo que nos formemos imágenes favorables o desfavorables de nuestros interlocutores en base a sus expresiones faciales, movimientos del cuerpo, tono de voz, mirada, uso del espacio, entre otros aspectos no verbales de la comunicación (Remland, 2003).

En lo que se refiere a la relación que se establece entre la comunicación no verbal y la enseñanza del inglés como LE, se destaca la idea de que, si se refuerza el trabajo con los signos de la CNV y se transmiten con viveza y energía, se produce una mejora en el rendimiento de los estudiantes y en su capacidad para memorizar el objeto no verbal de estudio. Según Sutiyatno, son tres las competencias no verbales que los profesores deben tener en cuenta en el proceso de enseñanza del inglés como LE:

a. Quinésicas:

○ Expresiones faciales: que se fundamentan en seis expresiones básicas universales -enfado, miedo, tristeza, disgusto, sorpresa y felicidad-. 
Artículo. Pablo Sánchez Abascal. "Tendencias en los estudios sobre comunicación no verbal: convergencias y divergencias."

- Contacto visual: el cual puede expresar interés, atracción, intimidad, dominancia, persuasión, agresividad o credibilidad.

○ Gestos: con los que se pueden reforzar una idea o describir algo.

b. Paralingüísticas: con el que se puede romper la dinámica de una clase atrayendo la atención de los estudiantes a las variaciones de timbre, tono, velocidad, etc.

c. Proxémicas: a través del cual se puede reforzar la relación y el vínculo que se establece entre profesores y estudiantes mediante un acercamiento adecuado o un posicionamiento dinámico en el aula.

En el apartado de conclusiones, Sutiyatno destaca que la enseñanza del inglés como LE debe basarse en el refuerzo explícito de las aproximaciones interculturales de los estudiantes, es decir, de sus propios principios culturales y de los principios de la cultura de la lengua de estudio.

\subsection{Non-verbal communication: why we need it in foreign language teaching and how we can Foster it with drama activities (2014)}

Artículo publicado en la revista lingüística Scenario en el que Surkamp profundiza sobre el papel de la enseñanza de la CNV en el desarrollo de la competencia comunicativa de los estudiantes de LE. Surkamp señala que las interacciones sociales no solo se producen a través de la comunicación verbal, sino que los signos no verbales que se realizan con todo el cuerpo representan un importante motor para la transmisión de significado.

Para la clasificación de los componentes no verbales que intervienen en los procesos de comunicación, Surkamp presenta un esquema en el que recogen sus principales componentes de estudio: 


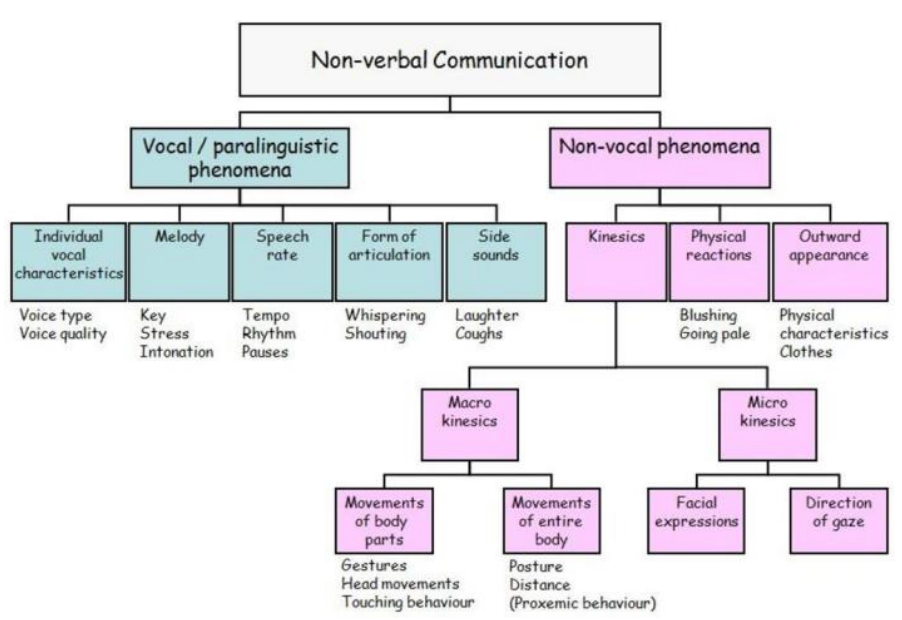

Figura 2. Vista de las principales formas de comunicación no verbal.

En este esquema se destacan dos grupos fundamentales de componentes no verbales. Por una parte, los elementos vocálicos paralinguiísticos, es decir, los elementos característicos vocales como la melodía, la velocidad del discurso, la forma de articulación o los sonidos como la risa o la tos, y por otra, los elementos no vocálicos relativos al sistema quinésico, a las reacciones físicas, al aspecto y a las expresiones faciales. En estos últimos, el subgrupo quinésico se subdivide, a su vez, en micro y macro quinésico. El macro quinésico incluye elementos como los movimientos del cuerpo (gestos, movimientos de cabeza, contacto físico) y los movimientos del cuerpo entero (postura, distancia social y comportamiento proxémico). El micro quinésico incluye elementos como las expresiones faciales o la dirección de la mirada. En lo que se refiere al componente proxémico, Surkamp destaca que la distancia apropiada para el desarrollo de una conversación depende de la situación social y de la cultura en la que tenga lugar. En este esquema no se incluyen los elementos relacionados con el concepto o el uso del tiempo (sistema cronémico) ni las características de los elementos del sistema cultural.

Surkamp expone la idea de que, si se abandona el estudio de los componentes no verbales en la enseñanza de LE, se pueden crear situaciones artificiales que no reflejen el transcurso real de los encuentros interculturales que tienen lugar en las interacciones comunicativas. De esta manera, los estudiantes no estudian de forma coordinada la palabra y la acción, el lenguaje y los gestos, como tampoco el propio proceso de producción en LE. 
Artículo. Pablo Sánchez Abascal. "Tendencias en los estudios sobre comunicación no verbal: convergencias y divergencias."

Uno de los objetivos fundamentales de este trabajo es el de expresar que los mayores obstáculos para el éxito de un intercambio comunicativo intercultural radican, frecuentemente, en errores no lingüísticos. Además, estos desencuentros interculturales producen una profunda ruptura comunicativa y son percibidos como comportamientos inapropiados e inadecuados. De esta manera, Surkamp expone algunos recursos para la introducción de la comunicación no verbal en el trabajo de la competencia escrita de LE. Para ello señala, entre otros, el uso de marcadores escritos como la literalidad que puede proporcionar la letra cursiva o el énfasis expresivo que produce el uso de las comillas. En relación con la enseñanza del componente no verbal a través de actividades de dramatización en la clase de LE, se propone el uso complementario de materiales visuales como fotografías, películas o audios mediante diferentes aproximaciones metodológicas: caminar de forma libre por la clase adaptando movimientos, jugar al espejo (gestos y expresiones faciales), adivinar profesiones mediante pantomimas o adivinar emociones o identificar tópicos u otras situaciones sociales comunes.

Finalmente, Surkamp señala que el aprendizaje intercultural mediante actividades de dramatización en la clase puede ser especialmente efectivo cuando los estudiantes toman conciencia de las propias características de la realización de gestos en su cultura de origen y, después, los comparan con su producción en la cultura de la lengua de estudio.

\subsection{Inventario de signos no verbales españoles y alemanes: estudio comparativo (2014)}

Estudio publicado en Red Electrónica de Didáctica del Español como Lengua Extranjera en el que Ascaso Fernández lleva a cabo un análisis de carácter comparativo entre algunos de los signos quinésicos más usados en la cultura española y alemana. Con el objetivo de realizar un inventario de signos no verbales básicos, Ascaso Fernández parte de una descripción pormenorizada de las teorías comunicativas de Cestero Mancera (1999) y Poyatos (1994a, 1994b) en la que se clasifican los signos que conforman la comunicación no verbal en los sistemas primarios (quinésicos y paralingüísticos) y secundarios (proxémicos y cronémicos), añadiendo además información sobre sus determinados usos funcionales en los procesos de interacción comunicativa. De esta manera, se señala que las funciones de los signos no verbales en los actos comunicativos se pueden clasificar en añadir información ya sea confirmando, debilitando o contradiciendo contenido-, comunicar -sustituyendo al 
lenguaje verbal o aportando información para hacerlo más significativo-, regular, organizar o estructurar la interacción, subsanar deficiencias verbales, o intervenir en conversaciones simultáneas.

Este estudio describe la triple estructura básica de la comunicación ${ }^{3}$ en la que se clasifican los signos no verbales en sistemas no verbales básicos -profundizando en los signos no verbales quinésicos y paralingüísticos-, y en sistemas de comunicación no verbal culturales, -refiriéndose, entre ellos, a los signos cronémicos y proxémicos-. Ascaso Fernández destaca, también, la importancia de enseñar una lengua meta de estudio y reconocer el resto de los recursos audibles y visuales que participan y acompañan al lenguaje verbal en los procesos de intercambio comunicativa. En este sentido, tal y como señala Cestero Mancera (1999), los estudios sobre los signos de comunicación no verbal se encuentran todavía en una fase experimental y de clasificación y recomienda la elaboración de inventarios comparativos interculturales que puedan conducir a la profundización en esta área de estudio. También según Cestero Mancera, el desarrollo de las investigaciones sobre la CNV debe llevarse a cabo de forma escalonada, progresiva y nivelada proponiendo su análisis en base a los niveles básicos expuestos en el Marco Común de Referencia para las Lenguas: aprendizaje, enseñanza, evaluación (2002). Ascaso Fernández presenta una propuesta metodológica para la introducción de los signos no verbales en la enseñanza de LE:

a. Presentar a los estudiantes de manera explícita o implícita los signos no verbales con los que se desea trabajar, su forma de producción y su función comunicativa.

b. Realizar actividades para ejercitar el uso de los signos no verbales tanto de forma individual como en pequeños grupos. Dichas actividades deben ser dirigidas por el profesor, cerradas, y pueden tener como tarea la interpretación, la producción de conversaciones, la creación de relatos y la observación participativa.

\footnotetext{
${ }^{3}$ Estructura basada en la interrelación entre el lenguaje verbal, la paralingüística y la quinésica (Poyatos, 1994a).
} 
Artículo. Pablo Sánchez Abascal. "Tendencias en los estudios sobre comunicación no verbal: convergencias y divergencias."

c. Realizar actividades dirigidas por el profesor y semi-cerradas para reforzar el aprendizaje de los signos no verbales en interacciones de dos estudiantes o en pequeños grupos.

d. Realizar actividades semi-dirigidas por el profesor y abiertas para propiciar la adquisición de los signos no verbales en interacciones más naturales y espontáneas.

Con el fin de crear un inventario completo comparativo de signos no verbales básicos españoles y alemanes, Ascaso Fernández aplica las recomendaciones de Cestero Mancera (1999) para la clasificación de los signos no verbales en base a sus usos sociales, estructuradores del discurso o comunicativos. Además, incluye en cada una de las fichas la descripción física del gesto, el signo paralingüístico que lo acompaña y, en los casos necesarios, la información proxémica y cronémica pertinente ${ }^{4}$.

\subsection{Nonverbal teacher-student communication in foreign language classroom (2014)}

Artículo de investigación publicado en la revista internacional Theory and Practice in Language Studies en el que la profesora Pan profundiza sobre el papel que juega el componente no verbal en la relación que se establece entre los estudiantes y los profesores en el aula, concretamente, en el ámbito de la adquisición del inglés como LE. Uno de los postulados fundamentales del estudio hace referencia al hecho de que los seres humanos hablamos a través de la articulación de los órganos vocales, aunque, para poder interactuar conversacionalmente, se necesita el apoyo de todos los recursos que nos ofrece el cuerpo, haciendo referencia a la pluralidad de signos no verbales que se transmiten durante todo el proceso del intercambio comunicativo. Este trabajo, cuyo objeto concreto de estudio es el comportamiento de los elementos proxémicos -la distancia personal y la orientación espacial-y los quinésicos de la comunicación no verbal, difiere de otros estudios centrados en CNV en la particular terminología que usa para clasificar los sistemas que lo conforman.

\footnotetext{
${ }^{4}$ No todos los signos quinésicos se acompañan necesariamente de signos complementarios no verbales de otros sistemas (Cestero Mancera, 1999).
} 
De hecho, existe cierta unanimidad en los estudios sobre $\mathrm{CNV}$, aunque con matices particulares según la perspectiva de estudio de cada línea de investigación, en partir de una clasificación básica de los sistemas no verbales en base a los signos quinésicos, paralingüísticos, cronémicos, proxémicos y al propio sistema de signos cultural de la lengua de estudio. Sin embargo, el trabajo de Pan, aunque sin variaciones drásticas con respecto a la citada clasificación, nombra los siguientes tipos de sistemas de comunicación no verbal ${ }^{5}$ :

a. Lenguaje corporal (movimientos del cuerpo, posturas, movimientos de la cabeza, expresiones faciales, gestos, entre otros).

b. Paralenguaje (características vocales, emoción, estilo del habla, características prosódicas como el ritmo, la entonación o el estrés).

c. Objeto de comunicación (ropa, corte de pelo, aspecto físico, entre otros)

d. Contexto psicológico (muebles, decoración interior, iluminación, colores, temperatura, ruido, música, aspectos proxémicos, entre otros).

Pan defiende en su trabajo que la posición o colocación espacial en la que el profesor imparte la clase en el aula (central y estática en un atril o dinámica y móvil entre las mesas y los estudiantes) incide directamente en la relación que el profesor establece con los estudiantes y, asimismo, en el proceso de aprendizaje de LE.

\footnotetext{
${ }^{5}$ El estudio de Pan (2014) usa una terminología alternativa para referirse a los mismos sistemas no verbales que se nombran en otros estudios. Sin embargo, el sistema cronémico no se nombra en ningún caso.
} 
Artículo. Pablo Sánchez Abascal. "Tendencias en los estudios sobre comunicación no verbal: convergencias y divergencias."
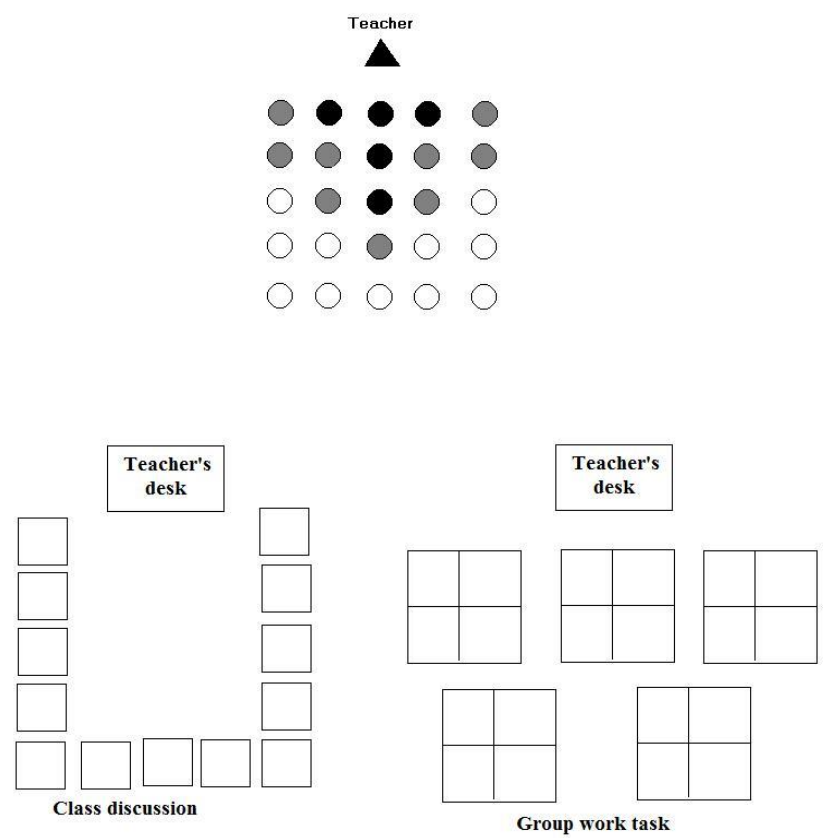

Figura 3. Disposición en el aula y posición del profesor.

Además, se pone también de manifiesto que la distribución del mobiliario en el aula, así como el consecuente posicionamiento espacial del profesor, influye en el modo en el que se comunican estudiantes y profesores y, por tanto, en el modo en el que se desarrolla el proceso de interacción comunicativa. Una posición clásica en la que el profesor se mantiene estático al frente de los estudiantes, como la que se recoge en la primera figura del cuadro 3 , puede conducir a impedimentos en el contacto físico (proxémicos) así como a obstaculizar el contacto visual con una parte de los estudiantes. Por otra parte, una distribución del mobiliario en forma de "U" o en pequeños grupos de trabajo, como en las figuras 2 y 3 del mismo cuadro 3, puede favorecer el contacto cercano del profesor con los estudiantes y, así pues, un acercamiento, una mayor accesibilidad comunicativa y la creación de una dinámica de trabajo en el aula que facilite el tratamiento de los signos no verbales que forman parte de los procesos de interacción comunicativa.

\subsection{La comunicación no verbal en el aula: un estudio de quinésica bajo la perspectiva metodológica Focus on Form (2014)}

Estudio presentado en la Universidad Antonio de Nebrija y desarrollado en el marco de los procesos gramático-cognitivos de aprendizaje de LE en la que se profundiza en las 
posibilidades que ofrece la aplicación de la perspectiva metodológica Atención a la Forma (Long y Robinson, 1998) para la enseñanza-aprendizaje de algunos signos quinésicos no verbales.

El trabajo parte del marco teórico de los estudios desarrollados por Poyatos (1994) y Cestero Mancera en el área de los signos no verbales de la comunicación $(1999,2004)$ y de los ejemplos didácticos para el estudio de los signos quinésicos que se recogen del diccionario o inventario gestual de las investigadoras Coll, Gelabert y Martinell (1990). A partir del análisis de dicho trabajo, se elabora una secuencia de actividades didácticas orientadas a la enseñanza de los signos no verbales quinésicos en relación con la orientación metodológica de la gramática cognitiva y su concreción en el formato Atención a la Forma. En base a las orientaciones didácticas dadas por Cestero Mancera (2004) para el trabajo con los signos de la CNV, las actividades que se presentan en este estudio siguen las siguientes recomendaciones metodológicas:

a. Presentación, explícita o implícita, de los signos no verbales, mostrando de forma clara y precisa los signos con los que se quiere trabajar.

b. Realización de actividades encaminadas al aprendizaje de los signos no verbales, dirigidas por el profesor y cerradas, en las que los estudiantes practiquen con diferentes actividades, tanto individuales como grupales.

c. Realización de actividades dirigidas a la adquisición de los signos no verbales, en las que el profesor semi-dirige actividades abiertas para la práctica de forma natural y espontánea.

Este estudio profundiza en la composición de los signos del sistema quinésico y en la clasificación de los elementos no verbales que lo conforman. Para ello, se presenta una clasificación de los signos quinésicos basada en parámetros y aspectos espaciotemporales, interlocucionales y lingüísticos, y en la propuesta de Ekman y Friesen (1969), recogida posteriormente por Knapp (1980), en la que se definen signos lingüísticos como los emblemas, los ilustradores, los reguladores, los signos expresivos-afectivos y los adaptadores. Más recientes son las referencias que se hacen a la clasificación de Poyatos 
Artículo. Pablo Sánchez Abascal. "Tendencias en los estudios sobre comunicación no verbal: convergencias y divergencias."

(1994a) en la que se señala que los signos quinésicos pueden ser, en relación a su forma de ejecución, libres o trabados, o la clasificación que hace Cestero Mancera (2004) en la que el sistema de signos quinésicos se subdivide en tres categorías interconectadas entre sí -los gestos o movimientos faciales y corporales, las maneras o formas convencionales de realizar las acciones o movimientos y las posturas o posiciones estáticas, resultantes, o no, de la realización de ciertos movimientos-.

En conclusión, este estudio pone en práctica una metodología experimental para la enseñanza de los signos no verbales quinésicos en el aula de LE bajo el formato de enseñanzaaprendizaje Atención a la Forma. Para ello se llevan a cabo sesiones de clase en las que se trabajan con diez signos quinésicos seleccionados para tal fin. Para el sistema de medición del grado de aprendizaje que se produce en los dos grupos que forman parte del estudio, uno control y otro experimental, se hace uso de dos tipos de prueba (un pretest y un postest) con los que se analiza cualitativa y cuantitativamente el nivel de conocimiento de dichos signos quinésicos.

\subsection{La comunicación no verbal en el aula: una propuesta de clasificación y análisis de gestos para la clase de E/LE (2013)}

La investigación llevada a cabo por Monterubbianesi y publicada en la revista didáctica MarcoELE, tiene como punto de partida la definición del concepto de cultura que propone Poyatos (1994a). A partir de dicha definición, se lleva a cabo una exhaustiva clasificación de las diferentes funciones lingüísticas que los gestos quinésicos pueden tener en las interacciones conversacionales. En el apartado teórico de este trabajo, Monterubbianesi expone que la clasificación de los signos quinésicos debe realizarse en consonancia a la función que cumplen en el propio acto comunicativo. De esta manera, se hace también referencia a varios conceptos que resaltan la importancia de dichas funciones comunicativas: las funciones lingüísticas de Knapp (1980), el intercambio verbal/no verbal de Poyatos (2006), o las categorías funcionales para la didáctica de la CNV de Cestero Mancera (1998).

Monterubbianesi señala también la importancia de las características de los signos no verbales quinésicos y su específica y particular realización en diferentes culturas. Así pues, define la gestualidad de los italianos como la expresión de una vida vivida entre aldeas y con una estructura familiar clara o definida. De los gestos de los judíos europeo dice que, bajo 
condiciones de dificultad durante una parte de su vida, son evasivos y de agresión localizada al más próximo. Además, los gestos utilizados de forma inconsciente en ambas lenguas expresan el pasado de ambas culturas y la complicada situación en la que llegaron a Norteamérica.

Monterubbianesi clasifica los gestos no verbales en relación con las funciones lingüísticas que estos pueden poseer en el desarrollo discursivo sean estos gestos que acompañan las palabras añadiendo informaciones -confirman, refuerzan, debilitan, contradicen, camuflan-, gestos que sustituyen a palabras -representan a las palabras, por deficiencia verbal, permiten conversaciones simultáneas- o gestos reguladores en la interacción -acompañan a las palabras, sustituyen a las palabras-.

Monterubbianesi recoge la idea de Cestero Mancera (2004) de elaborar un inventario de gestos quinésicos mediante la composición de fichas didácticas que, posteriormente, se puedan trabajar en el aula de LE. Finalmente, y con el objetivo de hacer de estas fichas un instrumento didáctico útil y práctico, se incluyen también los parámetros complementarios de categoría y subcategoría gestual, fuente, contexto, personaje, aplicación didáctica y comentarios que enriquecen aún más la explicación formal y funcional de dichos signos quinésicos.

\subsection{La comunicación sin palabras. Estudio comparativo de gestos usados en España y Brasil (2012)}

Esta tesis doctoral desarrollada por Nascimento Dominique constituye un análisis profundo en el área de la investigación de signos quinésicos entre dos lenguas y culturas diferentes. En este trabajo, Nascimento Dominique destaca el hecho de que hablar sin hacer uso de los elementos no verbales es prácticamente imposible y, si lo intentáramos, además de requerir un esfuerzo consciente, resultaría totalmente artificial. Por esta razón, en el estudio de los procesos de interacción comunicativa se debe atender, no solo a lo que decimos, sino a cómo lo decimos y a cómo nos movemos. El estudio tiene un doble objetivo de investigación: por un lado, descubrir y presentar las diferencias y similitudes en el repertorio de los signos no verbales entre hablantes españoles y brasileños y, por otro, analizar detalladamente el conjunto de emblemas gestuales usados en ambos países, valiéndose para tal fin de una serie de fichas pedagógicas ordenadas de acuerdo con su 
Artículo. Pablo Sánchez Abascal. "Tendencias en los estudios sobre comunicación no verbal: convergencias y divergencias."

función comunicativa. La investigación parte de un análisis de los términos de cultura, comunicación e interacción haciendo sendas referencias a definiciones de los tres conceptos. Para acercarse al término cultura, Nascimento Dominique se refiere a dicho término como «la unión de fenómenos, emociones y conductas de una comunidad». Destaca además la mención al modelo interdisciplinar de los culturemas que posibilita el análisis y la clasificación de la unidad mínima cultural: el culturema (Poyatos, 1994a). En lo concerniente a la comunicación humana, y partiendo de la afirmación de que el estudio formal siempre ha dado primacía a las palabras frente a lo no verbal, se señalan los estudios de Birdwhistell (1970) o Davis (1973) en los que se destaca la investigación sobre el efecto de los signos no verbales en el acto holístico de la comunicación humana. De esta forma, resalta la importancia de los mensajes simultáneos que tienen lugar durante la interacción comunicativa y que pueden influir en el propio acto comunicativo: gestos, posturas corporales, el tono de voz, etc. En cuanto a la CNV, Nascimento Dominique enfatiza la propia controversia del término no verbal entendiendo la negación explícita que implica la terminología communication minus language = non verbal communication . Nascimento Dominique realiza en este apartado un estudio pormenorizado de varias definiciones sobre la comunicación no verbal (Ekman y Friesen, 1969; Knapp, 1980; Poyatos, 1994a, et. al) así como del desarrollo histórico de este concepto desde los estudios iniciales de Darwin (1872). También recoge la clasificación de autores como Ekman y Friesen (1969) o Cestero Mancera (1999) en las que se clasifican la plurifuncionalidad de los signos de los sistemas de la CNV apuntando, de forma aislada o conjunta, sus funciones fundamentales: añadir información al mensaje verbal o matizarlo, sustituir el mismo mensaje verbal, regular la interacción, corregir posibles fallos verbales o posibilitar las conversaciones simultáneas.

Nascimento Dominique destaca también que la interacción comunicativa se basa en un complejo entramado de signos y sistemas culturales, verbales y no verbales sin los cuales no se puede transmitir un mensaje de forma efectiva. Así pues, e interesándose por todos aquellos elementos no verbales que puedan generar e intervenir en la interacción conversacional, organiza y categoriza los signos no verbales en sistemas básicos (verbal, paralingüístico y quinésico), sistemas culturales (cronémico y proxémico), sistemas físicos y signos culturales. 
Para terminar, el estudio presenta el corpus del citado inventario en forma de fichas comparativas y explicativas en relación con la clasificación de los usos y las funciones comunicativas de los gestos no verbales de Cestero Mancera (1999).

\subsection{La comunicación no verbal y su relación con la interpretación (2012)}

El artículo desarrollado por la traductora Valiente Boloy y publicado en la Revista de la Asociación Cubana de Traductores e Intérpretes (ACTI), analiza la relación existente entre los sistemas quinésico y paralinguiístico de la CNV con los procesos interculturales de traducción e interpretación. Para ello, analiza la forma en la que ambos sistemas pueden contribuir a facilitar la comprensión del mensaje emitido y mejorar, de esta forma, la calidad del proceso de interpretación comunicativa.

El marco teórico del trabajo realiza un recorrido histórico por algunos de los estudios realizados en el siglo XX en el área de la CNV haciendo hincapié en el notable impulso que supuso la sistematización metodológica del abordaje de los procesos de interacción comunicativa en las décadas de los años sesenta y setenta. Según Valiente Boloy, la CNV adquiere una importancia primaria en los procesos de interpretación y considera que el intérprete que no perciba o comprenda este tipo de comportamiento tendrá una imagen distorsionada o inexacta de la situación en la que debe operar (Collados Aís, 1998). De esta manera, el conocimiento del lenguaje no verbal de la parte foránea involucrada en el acto comunicativo es de gran utilidad entendiendo el proceso de observación de la propia cultura como punto de partida para, después, poder comparar estos elementos con la cultura extranjera. En relación con dicha observación de la cultura extranjera, Valiente Boloy defiende que el intérprete debe ser capaz de elaborar un método de observación y autoobservación de actitudes, conductas, estilos comunicativos y vivencias, y así ser consciente de que la imagen que tenemos de otra persona está filtrada por nuestras experiencias y nuestra propia cultura.

El estudio de Valiente Boloy analiza y describe con detalle los sistemas y signos culturales que conforman la CNV y ahonda, concretamente, en los dos sistemas en los que pone su foco de atención: el sistema quinésico y el sistema paralingüístico. Además, pone en relación ambos sistemas con el complejo proceso de traducción e interpretación y señala que el AIIC (Code for the Use of New Technologies) resalta la importancia del campo visual del 
Artículo. Pablo Sánchez Abascal. "Tendencias en los estudios sobre comunicación no verbal: convergencias y divergencias."

intérprete y la CNV para una correcta interpretación. En esta misma línea, el estudio señala que el intérprete no debe conocer solo el idioma con el que trabaja sino otros elementos relacionados tales como la cultura, el medio y los elementos no verbales que intervienen en los intercambios comunicativos.

Para terminar, Valiente Boloy pone de manifiesto que un proceso completo de traducción e interpretación debe atender a la totalidad de aspectos verbales y no verbales que actúan e interactúan de forma simultánea en un intercambio comunicativo. De esta manera, y entre otros aspectos, se menciona la importancia de la apariencia del interprete -debe ser neutral y el vestuario acorde a la situación en la que se produzca-, la postura -de manera cómoda-, la relación de la comunicación entre el intérprete y el emisor, la relación de la comunicación entre el intérprete y el público receptor, u otros aspectos importantes como la entonación, el tono de voz, sonidos como la risa o las interjecciones, o las pausas que toma el emisor. Como indica Valiente Boloy, todos esos elementos influyen en el complejo proceso de interpretación de los mensajes y, por ende, en los propios intercambios comunicativos que se producen durante la traducción e interpretación de una LE.

\section{Tendencia: convergencias y divergencias de los estudios}

Los trabajos seleccionados para la elaboración de este estudio son una solo muestra del notable incremento que han experimentado los trabajos sobre el funcionamiento de los signos no verbales en las interacciones comunicativas en los últimos años. Asimismo, y debido a la propia naturaleza multidisciplinar de los signos de la CNV, se refleja la variedad sistémica en el tratamiento de los denominados signos no verbales afrontando el estudio de dichos signos desde perspectivas muy diferentes, si bien complementarias al mismo tiempo en muchos de los casos.

El objetivo fundamental de este estudio es la contextualización y delimitación de las teorías y prácticas metodológicas que se aplican en la investigación de los signos no verbales. En esta línea de acción, los estudios seleccionados permiten trazar algunas conclusiones sobre las convergencias o divergencias que se desprenden de la aplicación metodológica en cada uno de los trabajos, del sistema/sistemas no verbales sobre los que investigan o de su aplicación didáctica que puede derivar en los procesos de enseñanza-aprendizaje. También son relevantes los datos cuantitativos que hacen referencia al número de estudios que abordan 
el sistema quinésico, paralingüístico, proxémico, cronémico, cultural, o una forma combinada de ellos, ya que muestran una tendencia en la frecuencia de estudio de determinados sistemas. En los aspectos referidos a la frecuencia de estudio y a los sistemas no verbales más analizados cabe destacar que:

a. Son notablemente recurrentes los estudios que analizan el uso y el funcionamiento de los signos no verbales quinésicos. De esta manera, 9 de los 10 estudios seleccionados abordan las características funcionales de los signos quinésicos y el papel que juegan en los procesos de enseñanza de LE y en las interacciones conversacionales. Sin embargo, se pueden constatar perspectivas metodológicas que afrontan los gestos, los movimientos y las posturas desde posicionamientos teóricos diferentes. En cualquier caso, dichas perspectivas no implican la unilateralidad en el estudio del sistema quinésico y la complementariedad de muchas de sus perspectivas se manifiesta en el estudio de otros de los sistemas no verbales. Los trabajos que se presentan en este estudio investigan el uso y el funcionamiento de los signos quinésicos a través de aplicaciones metodológicas que abarcan áreas múltiples de estudio. Valga de ejemplo el siguiente listado:

El aprendizaje del componente no verbal a través de herramientas virtuales (Méndez Guerrero, 2016); El papel de la enseñanza-aprendizaje de la CNV y de los signos quinésicos en LE a través de actividades de dramatización (Surkamp, 2014); La CNV y el uso de la gestualidad en la enseñanza del inglés como LE (Sutiyatno, 2015); La interpretación del signo quinésico del silencio en los procesos de interacción conversacional (Méndez Guerrero, 2016); Estudios comparativos interculturales centrados en el estudio de los signos quinésicos (Nascimento Dominique, 2012; Ascaso Fernández, 2014; Sánchez Abascal, 2014); La elaboración de inventarios de signos y gestos no verbales básicos (Nascimento Dominique, 2012; Ascaso Fernández, 2014); La CNV entre profesor y estudiante en el aula de LE y el rol de la gestualidad en el proceso didáctico (Pan, 2014; Sutiyatno, 2015); La enseñanza de los signos quinésicos bajo la perspectiva de estudio Atención a la Forma (Sánchez Abascal, 2014); Clasificación de las funciones lingüísticas de los signos quinésicos (Monterubbianesi, 2013); Los 
Artículo. Pablo Sánchez Abascal. "Tendencias en los estudios sobre comunicación no verbal: convergencias y divergencias."

signos no verbales, también quinésicos, y su relación con la traducción y la interpretación (Valiente Boloy, 2012). En lo relativo al carácter multidisciplinar de los estudios sobre los signos de la CNV y al destacado posicionamiento que parece haber tomado el análisis del uso y la función comunicativa de los signos quinésicos se debe resaltar que, en la mayoría de los casos, los trabajos abordan no solo el estudio del sistema quinésico de forma aislada sino mediante la combinación con el análisis de otros signos no verbales que forman parte de los procesos de la interacción conversacional. De esta manera, en el estudio de los signos quinésicos -ya sean los gestos, las posiciones, el silencio o la mirada- se entrelazan los unos con los otros a la vez que se solapan con el análisis de las distancias sociales en el aula (proxémica), las funciones lingüísticas (CNV) o las prácticas didácticas para su enseñanza en el aula de LE. Esta riqueza de perspectivas metodológicas es una muestra de la pluralidad de aspectos y factores que se pueden investigar en un área de acción caracterizada por su amplia divergencia temática y por su incidencia tanto en los procesos de aprendizaje de LE como en los propios procesos de interacción comunicativa.

b. En el extremo opuesto respecto a la recurrente frecuencia de estudios sobre los signos no verbales quinésicos, encontramos una marcada escasez de trabajos que analizan -ya sea de forma directa o transversal- el sistema no verbal cronémico, es decir, el sistema que estudia las conceptualización lingüística y cultural de los aspectos temporales ${ }^{6}$. De esta manera, solo 1 trabajo de los 10 analizados (Méndez Guerrero, 2016) ahonda en el funcionamiento y en las características del sistema cronémico con la particularidad añadida de referirse a un estudio holístico que estudia los signos de la CNV en conjunto y a través de herramientas virtuales. Esto puede ser una muestra significativa de la escasez de trabajos que se orientan en la aplicación concreta de análisis de los signos cronémicos y, por tanto, de la falta de estudios linguiísticos que profundizan en la conceptualización temporal a través de la lengua y sus referentes culturales.

\footnotetext{
${ }^{6}$ Como indica Sánchez Abascal (2014), no se concibe de la misma manera el tiempo de espera en la expresión vuelvo en 5 minutos en todas las lenguas y culturas.
} 
c. Algo similar a lo que ocurre con el estudio de los signos del sistema cronémico se detecta en los artículos que tratan el sistema de los signos no verbales culturales. Así pues, solo 2 de los 10 trabajos analizados profundizan en el funcionamiento y en las características de dicho sistema (Surkamp, 2014; Méndez Guerrero, 2016) centrado en la investigación sobre la conceptualización de las tendencias culturales como el modo de vestir, los cortes de pelo, la forma de caminar, etc. Además, y al igual que ocurriera con el sistema no verbal cronémico, el análisis de los signos culturales se hace de forma combinada al estudio de los sistemas quinésico, paralingüístico, proxémico y cronémico de la CNV. Por lo tanto, las mismas consideraciones que se desprenden de los estudios del sistema cronémico respecto a su escasez e interdependencia intersistémica se derivan de los trabajos que profundizan en el estudio del sistema no verbal cultural.

d. En un punto intermedio en la escala de frecuencia de los estudios que abordan los diferentes sistemas de la CNV se encuentran los trabajos que profundizan en el funcionamiento del sistema paralingüístico de la comunicación (5 de los 10 trabajos) y del sistema proxémico (4 de los 10 trabajos). Cabe destacar que, de todos ellos, ninguno analiza el funcionamiento de los sistemas paralingüístico o proxémico de forma aislada, sino que, a través de su combinación con el estudio de otros sistemas no verbales, analizan sus usos y sus características funcionales, principalmente con un fin didáctico. Resulta también importante destacar que el sistema de signos paralingüístico, en combinación con el sistema quinésico y el lenguaje verbal, constituye la triple estructura del lenguaje (Poyatos, 1994a) e indicar, de esta forma, que podría indicar la relativa recurrencia de su aparición en los trabajos analizados. De hecho, la triple estructura del lenguaje es considerara por diferentes investigadores como la base comunicativa de la mayor parte de las interacciones conversacionales. Por otra parte, es significativo la relativa recurrencia de estudio del sistema de signos proxémico en comparación con la escasez de trabajos sobre el otro sistema no verbal que conforman los denominados signos secundarios -el sistema cronémico-. Asimismo, y al igual que ocurriera 
Artículo. Pablo Sánchez Abascal. "Tendencias en los estudios sobre comunicación no verbal: convergencias y divergencias."

con los sistemas cronémico y cultural, su estudio se caracteriza por su interrelación e interdependencia con el resto de los sistemas de la CNV, unas veces de forma holística y otras de modo específico en combinación con uno solo de los sistemas de signos no verbales.

\section{Conclusión}

Más allá de las conclusiones de carácter porcentual que se pudieran obtener de la frecuencia de estudio de los diez trabajos seleccionados, se puede destacar que el predominante abordaje del sistema quinésico se ha convertido en una práctica generalizada en la mayoría de las investigaciones de los signos no verbales ${ }^{7}$. Dicha predominancia y riqueza de estudio del sistema no verbal quinésico sobre el resto de los sistemas se refleja, por ejemplo, al realizar búsquedas bibliográficas en línea basada en conceptos y términos genéricos como comunicación-no-verbal o signos-no-verbales. En muchos de estos casos, la búsqueda conduce a estudios especializados sobre los signos no verbales quinésicos y, más concretamente, sobre el análisis funcional de los gestos y de sus giros más usuales. Un claro ejemplo de ello son los dos trabajos que forman parte de este apartado -junto al estudio de Sánchez Abascal (2014) que parte del diccionario de gestos de Coll, Gelabert y Martinell (1990)- en los que se estudian de forma analítica y comparativa la producción y el significado de un determinado número de gestos usuales en dos lenguas diferentes (Nascimento Dominique, 2012; Ascaso Fernández, 2014).

La constatación de la recurrente insistencia en el estudio y en el análisis del sistema quinésico respecto al resto de los sistemas de la CNV conduce al planteamiento de preguntas en torno a la explicación y al porqué de dicha predominancia. Según Cestero Mancera (2004), este hecho podría deberse a que el sistema quinésico es el que está más claramente implicado en cualquier acto de la comunicación y en las interacciones conversacionales humanas. Además, la propia naturaleza fácilmente observable del sistema quinésico respecto al resto de los sistemas no verbales -realización física a través de gestos, posturas o posiciones-y, por consiguiente, su relativa accesibilidad en el propio proceso de recogida de datos puede

\footnotetext{
${ }^{7}$ Nascimento Dominique (2012) señala y anuncia que el sistema quinésico es el más estudiado de entre todos ellos y, a su vez, el más complejo.
} 
ser otro de los factores influyentes en el mayor volumen de estudios sobre este sistema. No por menos recurrentes, el resto de los sistemas no verbales dejan de estar bien acotados o definidos en su objeto de estudio. Sin embargo, una naturaleza menos observable, así como un proceso de recogida de datos más laborioso e instrumentalizado, hacen de ellos sistemas menos estudiados y, en la mayoría de los casos, referidos a su aparición simultánea con el sistema de los signos no verbales quinésicos.

Finalmente, se quiere señalar una de las conclusiones más destacadas que se deriva de las convergencias y divergencias de las investigaciones analizadas en este trabajo: la necesaria interrelación de todos los sistemas no verbales en el estudio de las interacciones comunicativas no verbales ya que se trata de una acción casi imposible, además de poco natural, el usar solo un determinado conjunto de signos no verbales sin poner en marcha el resto de signos quinésicos, paralingüísticos, cronémicos, proxémicos o culturales que matizan, acompañan o aportan parte del significado del mensaje que se quiere transmitir. Dicha conclusión, unida a las necesidades específicas de cada investigación, puede ser una premisa fundamental para la planificación y el desarrollo de los estudios socioculturales en el área de la CNV.

\section{REFERENCIAS}

Ascaso, Samanta. Inventario de signos básicos españoles y alemanes: estudio comparativo. 2014. Universidad Antonio de Nebrija, tesis.

Birdwhistell, Ray L. Kinesics and Context Essays on Body Motion Communication. University of Pennsylvania Press, , 1970.

Cestero Mancera, Ana M. "El estudio de la comunicación no verbal y su aplicación a la enseñanza de lenguas extranjeras". Estudios de comunicación no verbal. Edinumen, 1998, pp. 7-16.

---. "La interrupción en la conversación en lengua española". Lingüística para el siglo XXI, vol. I, Actas del III Congreso de Lingüística General de Salamanca, 1999, pp. 429-434.

---. "La comunicación no verbal". Vademécum para la formación de profesores: Enseñar español como segunda lengua/lengua extranjera, edición de J. Sánchez Lobato e I. Santos Gargallo. SGEL, 2004, pp. 593-616.

Coll, Josep, Maria José Gelabert y Emma Martinell. Diccionario de gestos con sus usos más usuales. Edelsa, 1990.

Collado Aís, Angela. La evaluación de la calidad en interpretación simultánea. La importancia de la comunicación no verbal. Comares-Interlingua, 1998.

Darwin, Charles. The expression of the emotions in man and animals (1872). Trad. esp.: La expresión de las emociones en los animales y el hombre. Alianza, 1984. 
Artículo. Pablo Sánchez Abascal. "Tendencias en los estudios sobre comunicación no verbal:

Davis, Flora. Inside Intuition: what we know about nonverbal communication. Mc Graw Hill, 1973.

Ekman, Paul y Wallace V. Friesen. "The repertoire of nonverbal Behavior: categories, origins, usage and coding". Semiotica, vol. 1, núm. 1, 1969, pp. 49-98.

---. "A new plan-cultural facial expression of emotion". Motivation and emotion, vol. 10, núm. 2, 1986, pp. 159-168.

Espinoza, Norelkys. "Criterios para la selección de la información científica odontológica en la world wide web". Act. Odont, vol. 41, núm. 3, 2003, pp. 251-257.

García, Dago. La comunicación no verbal en el aula de E/LE: consideraciones y propuesta de aplicación práctica. 2008. Universidad de Alcalá, tesis.

Knapp, Mark L. Essentials of nonverbal communication. Holt Rinehart \& Winston, 1980.

Long, Michael H. y Peter Robinson. "Focus on Form: Theory, research and practice". Focus on Form in second language acquisition, edición de C. J. Doughty y J. Williams. Cambridge University Press, 1998, pp. 15-41.

Monterubbianesi, María G. "La comunicación no verbal en en el aula: una propuesta de clasificación y análisis de gestos para la clase de E/LE”. Marcoele, núm. 16, 2013. https://marcoele.com/descargas/16/monterubbianesi-comunicacion_no_verbal.pdf

Consultado el 12 de febrero de 2021.

Moreno de los Ríos, Belén. Programación de cursos de lenguas extranjeras. Fundación Antonio de Nebrija, 1998.

Nascimento Dominique, Nilma. La comunicación sin palabras. Estudio comparativo de gestos usados en España y Brasil. Servicio de Publicaciones de la Universidad de Alcalá, 2012.

Nuñez, Mirta. "Criterios para la evaluación de la calidad de las fuentes de información sobre la salud en Internet”. Acimed, vol. 10, núm. 5, 2002.

Pan, Qi. "Nonvebal teacher-student communication in the foreign language classroom". Theory and practice in language studies, vol. 4, núm. 12, 2014, pp. 2627-2632.

Perdomo, Bexi. "Búsqueda y selección de textos en la internet para investigación científica". Saber, vol. 20, núm. 1, 2008, pp. 109-116.

Piderit, Theodor. Handleiding: tot der leer der gelaatkennis. Amsterdam, 1859

Poyatos, Fernando. La comunicación no verbal. Cultura, lenguaje y conversación. Istmo, 1994a.

---. La comunicación no verbal. Paralenguaje, kinésica e interacción. Istmo, 1994b.

---. "La enseñanza del español a extranjeros a través de los estudios de comunicación no verbal". Lingüística aplicada a la enseñanza del español como lengua extranjera: desarrollos recientes, edición de A. M. Cestero. Servicio de Publicaciones de la Universidad de Alcalá, 2006, pp. 27-46.

---. La comunicación no verbal en la enseñanza integral del español como lengua extranjera. Servicio de Publicaciones de la Universidad de Alcalá, 2017.

Terrón Blanco, Jose Luis. El silencio radiofónico. Universitat Autónoma de Barcelona, 1992. Sánchez Abascal, Pablo. La comunicación no verbal en el aula: un estudio de kinésica bajo la perspectiva metodológica Atención a la Forma. 2014. Universidad Antonio de Nebrija, tesis.

Remland, Martin S. Nonverbal communication in everyday life. Houghton Mifflin, 2003.

Spencer, Herbert. The principles of psychology. Londres, Longman, Brown, Green and Longmans, 1855. 
Surkamp, Carola. "Non-verbal communication: why we need it in foreign language teaching and how we can Foster it with drama activities". Scenario: Language, Culture, Literature, vol. III, 2014, pp. 12-17.

Sutiyatno, Sukris. "The role of nonverbal communication in English teaching". Jurnal Transformasi, vol. 11, núm. 1, 2015, pp. 27-32.

Valiente, Yadilka. "La comunicación no verbal y su interpretación”. Revista de la asociación cubana de traductores e intérpretes, núm. 2, 2012, pp. 4-12. 